\title{
IN SEARCH OF THE ELUSIVE TRUE SURFACE
}

\author{
Peter Z. Takacs, Karen Furenlid \\ Brookhaven National Laboratory, Upton, NY 11973 \\ and \\ Eugene L. Church \\ U. S. Army, ARDEC, Dover, NJ 07801-5001
}

August 1990

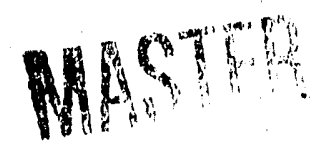

*This research was supported by the U. S. Department of Energy:

Contract No. DE-AC02-76CH00016.

PISTRIBLTION OF THIS DOCUMENT 


\section{DISCLAIMER}

This report was prepared as an account of work sponsored by an agency of the United States Government. Neither the United States Government nor any agency thereof, nor any of their employees, nor any of their contractors, subconitractors, or their employees, makes any warranty, express or implied, or asrumes any legal liability or responsibility for the accuracy, completeness, or usefulness of any information, apparatus, product, or process disclosed, or represents that its use would not infringe privately owned rights. Reference herein to any specific commercial product, process or service by trade name, trademark, manufacturer, or otherwise, does not necessarily constitute or imply its endorsement. recommendation, or favoring by the United States Government or any agency, contractor, or subcontractor thereof. 'The views and opinions of authors expressed herein do not necessarily state or reflect those of the United States Government or any agency, contractor or subcontractor thereof. 


\title{
IN SEARCH OF THE ELUSIVE TRUE SURFACE
}

\author{
Peter Z. Takacs, Karen Furenlid \\ Brookhaven National Laboratory, Upton, NY 11973 \\ and \\ Eugene L. Church \\ U. S. Army, ARDEC, Dover, NJ (07801-5001
}

\begin{abstract}
Measurement of an optical surface by a figure measuring device allows one to make an estimate of the shape of the surface. The absolute accuracy in locating the "true" surface depends on the sources of error in the measurement process - both random errors and systematic errors. Analysis procedures have been developed to extract surface figure information from optical profiler measurements on large mirrors. Rigid-body orientation effects are important in analyzing the shape of the off-axis ellipsoidal reflectors used as grazing incidence mirrors in soft-x-ray synchrotron instrumentation. Knowledge of the noise level and optical aberration function of the Long Trace Profiler allow us to place error bars smaller than $\lambda / 100$ on measurements of surfaces that are more than $500 \mathrm{~mm}$ long.
\end{abstract}

\section{INTRODUCTION}

Over the past several years the Optical Metrology Laboratory in the Instrumentation Division at Brookhaven National Laboratory has been involved in the measurement of the finish and figure optical surfaces used as reflectors for soft $x$-rays. The extreme grazing incidence angle required of the incident beam in most cases necessitates the use of highly aspheric off-axis segments of conic surfaces to produce an image. A typical mirror would be in the shape of a cylinder or toroid or ellipsoid with a length of $700 \mathrm{~mm}$, used at a $2^{\circ}$ grazing incidence angle. Such a mirror would have a minor radius of curvature on the order of $50 \mathrm{~mm}$ and a major radius on the order of several hundred meters. Such extreme aspheres are nearly impossible to test with conventional interferometers. We have developed a figure measuring instrument $[1,2]$ optimized for measuring the shape of cylindrical aspheres based on a scanning profiling interferometer. Profiling instruments are not used routinely in the optical shop for figure measurement. We are reporting on some data analysis techniques that are not usually encountered in conventional interferometric testing methods and on how we determine the ultimate performance of the measuring instrument. All of this is related to the question of how we determine what the "true" surface looks like.

\section{FIGURE BY PROFILOMETRY}

The use of profilometry to determine the figure of optical surfaces is not unknown in the optical shop. Ball spherometers represent a class of profiling device that is commonly used to determine the curvature of both ground and polished optical surfaces. What is not common is the use of scanning profilers to measure the figure of polished surfaces. Perhaps the reason for this has been the availability of full-aperture interferometer systems that can measure the reflected wavefront from an optical surface to a high degree of precision and the lack of available profiling machines with the required accuracy. Certainly for spheres or flats a full-aperture phase measuring interferometer test is extremely fast compared to the time required to measure the surface with a scanning optical or mechanical stylus system. However, there are certain measurements that can be done best by profilometry. Two of these are rapid measurement of aspheric optics and absolute radius of curvature measurement. We have developed a scanning optical profiling instrument, the Long Trace Profiler (LTP) $[1,2]$, which we routinely employ to measure the absolute shape of cylindrical aspheres and bent mirrors used in synchrotron radiation beamlines, as well as of conventional spherical

*This research was supported by the U. S. Department of Energy: Contract No. DE-AC02-76CH00016. 
and flat optical surfaces. Data analysis techniques used to interpret profiler data differ from those used to analyze fullaperture interferograms. We have encountered several interesting problems in interpreting profiler data and present some of our techniques here. "The goal of this work is to enable us to put limits on the true shape of an optical surface, knowing the limitations of the measuring system.

When we make a measurement of the profile of a surface, we are approximating the "true" surface profile. The measured profile can be decomposed into two parts: a low-order polynomial function and the residual which we call surface finish. This process is illustrated by the equation:

$$
Z_{\text {true }}\left(x_{i}\right) \approx Z_{\text {meas }}\left(x_{i}\right)=a_{0}+a_{1} x_{i}+a_{2} x_{i}^{2}+\ldots+Z_{\text {finish }}\left(x_{i}\right)
$$

The measured surface is only an approximation of the true surface and is itself composed of a polynomial, which we traditionally associate with "figure", and a residual function, which we identify "ilth surface finish. In conventional interferometer analysis programs the figure part is usually represented by Zernike polynomials and the finish part is disregarded or is converted to an RMS residual error number. We, however, are usually more interested in the residual finish term because it contains the mid-frequency surface roughness information that tells us what the near angle scattering properties of the mirror will be. Note that Zernike polynomials could also be replaced with a Fourier series or other complete orthonormal set of basis functions, or the figure term could be expressed in terms of ordinary polynomials as a function of the distance coordinate along the local coordinate axis. Ordinary polynomials are not orthogonal, however, which creates some matters of interpretation in the measured data. The use of simple polynomials to remove the lowest order surface terms is usually justified in profiler measurements because the lowest order terms physically correspond to piston, tilt, and curvature of the surface. For spherical surfaces it is usually sufficient to remove these three lowest-order polynomial terms and view the residual as finish.

The process of removing low-order terms from a set of data is called "detrending" and is a common practice in statistical analysis. If the surface being measured were perfectly made, then the detren ing process would be trivial. But real surfaces are not perfect, and detrending becomes a matter of interpretation. cilferent detrending methods can be used to emphasize different features of a surface. For instance, if it is desired to view the departure of a surface from a circle (note that real three-dimensional surfaces are viewed as two-dimensional sections by the profiler), then one needs to subtract from the raw data a second order least-squares-fit (LSF) polynomial. One could also ask what is the departure from a given radius of curvature. In that case one would have to constrain the value of the second order coefficient to the value corresponding to the desired radius - 1/(2R) - and then do the LSF. Or one might only be interested in the central region of the profile, away from any edge roll-off. Then one would have to restrict the range of the LSF to a subset of the data. If one is measuring an ellipsoid, one would probably need to include the third order term in the LSF to describe the ideal surface.

Various detrending options are illustrated in Fig. 1. A profile measurement was made on a fused silica toroid $300 \mathrm{~mm}$ in length with a desired major radius of 1500 meters. Fig. la is the "as-measured" height profile with piston and tht removed. (These measurements were made with the LTP which is really a slope measuring device. The slope data has been converted to height data for ease in interpretation.) One can readily see that the surface consists of ihree zones: a good quality central zone and edge roll-off at each end. The radius of curvature derived from a second order LSF to this height profile is $1378 \mathrm{~m}$, which is not too far from the desired $1500 \mathrm{~m}$. However, a second order LSF to the central region only, between $50 \mathrm{~mm}$ and $235 \mathrm{~mm}$, results in a radius of $943 \mathrm{~m}$, which is far from the desired result. If we wish to remove the average radius of the central zone and look at the residual departure from a circle, the result is shown in Fig. Ib. Here a radius of $986 \mathrm{~m}$ is removed from the slope data and the residual is plotted. Most of the curvature is removed from the central zone and the residual clearly shows the edge roll-off. There is a slight discrepancy between the two radius numbers, $943 \mathrm{~m} \mathrm{vs} .986 \mathrm{~m}$, the first computed from the height data and the second computed from the raw slope data. This difference in radius values probably arises because to the height data, the LSF is done with a second order polynomial after converting raw slope to height, while for the slope data, the LSF is done 

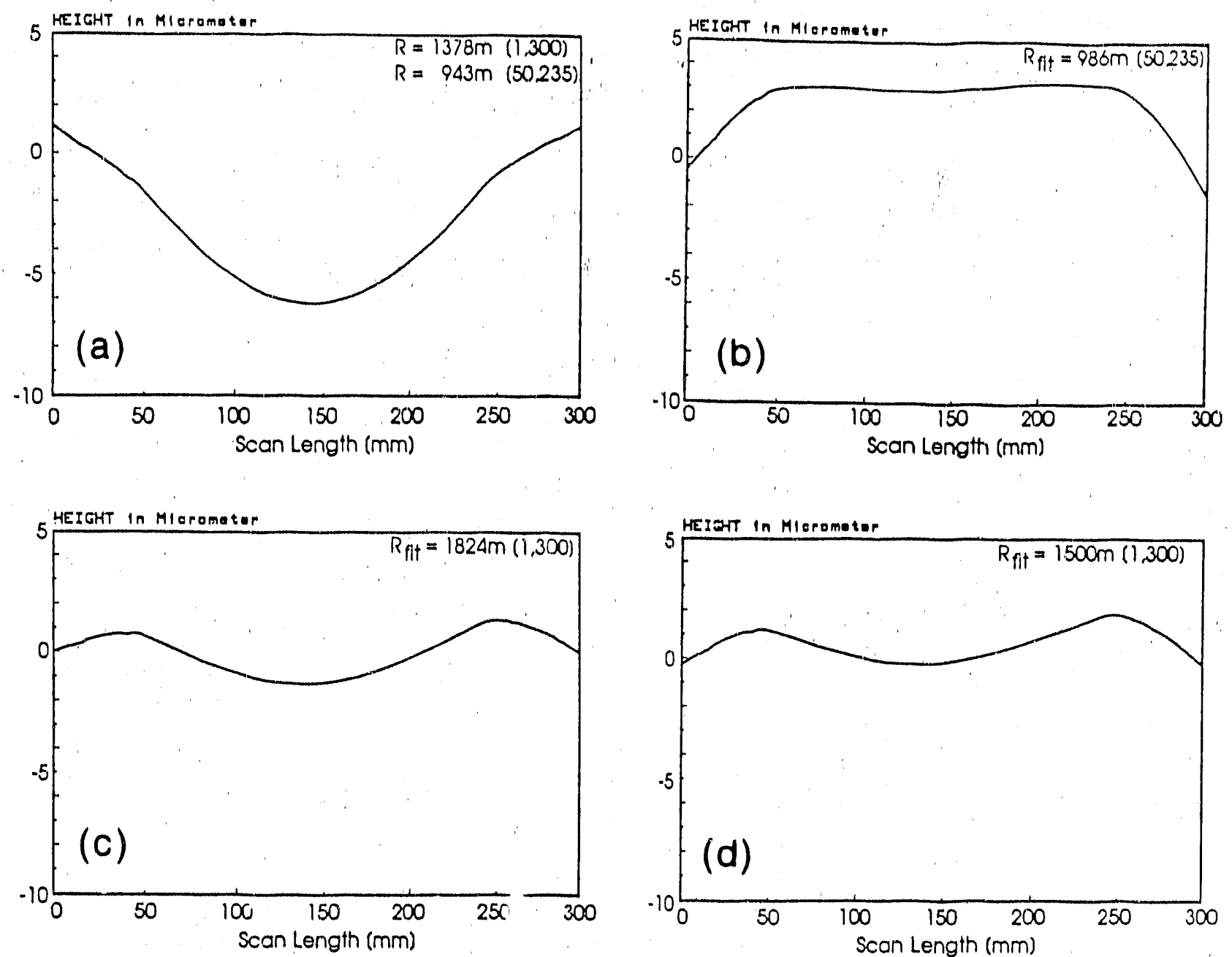

Figure 1.) Profile measurement of fused silica toroid with design radius $R=1500$ meters: a) Height computed from raw slope function. LSF radius values computed from height function over entire range $=300 \mathrm{~mm}$ length, and over central zone from 50 to $235 \mathrm{~mm}$. Edge roll-off is evident near each end of the profile. b) Residuai profile after best-fit radius to central zone, $R=986 \mathrm{~m}$, is subtracted from slope function hefore height calculation. c) Residual profile after subtraction of $R=1824 \mathrm{~m}$ least squares best fit radius term fit to slope data over full range; RMS $\sigma_{z}=0.838 \mu \mathrm{m}$. d) Subtraction of design radius $\mathrm{R}=1500 \mathrm{~m}$ from slope data. $\mathrm{RMS} \sigma_{\mathrm{z}}=0.885 \mu \mathrm{m}$ for this constrained radius is larger than unconstrained LSF value in (c).

with a first order polynomial to the slope data before converting to height. The data points are weighted differently in the fitting process between the two polynomials, resulting in a slight difference between the derived radius numbers. This discrepancy would not exist if the surface were mathematically ideal. The dependence of radius value on detrending method is more clearly illustrated in Fig. 1c, which is a plot of the residual surface after a first order LSF detrend was made to the full 300 point slope function, and then the height function computed by integration. The best-fit radius computed from the value of the first order coefincient in the slope detrend is $1824 \mathrm{~m}$, while the radius derived from the second order coefficient fit to the height profile in Fig. 1a is $1378 \mathrm{~m}$. Again, the probable cause for this difference is the different weighting of the data points in the LSF between the two data sets. The discrepancy is larger in the latter case because the entire da 4 set is used instead of only the central zone, and the departure of the surface from an ideal circle is more pronounced over the larger data range. 
For completeness, the design radius, $1500 \mathrm{~m}$, is removed from the slope function and the residual height is plotted in Fig. 1d. One can barely see a difference from Fig. 1t, which is the unconstrained LSF, but the RMS values of the residuals tell the story: the unconstrained LSF in Fig. 1c does indeed have the lower RMS value, $0.838 \mu \mathrm{m}$ versus $0.885 \mu \mathrm{m}$ for the $\mathrm{R}=1500 \mathrm{~m}$ constraint.

\section{RIGID BODY ORIENTATION EFFECTS}

A profiling instrument views the surface in a special way: the probe axis defines the local normal direction and the scan direction defines the orthogonal coordinate, which is the independent variable. We usually assume that the lowest point on the surface (the pole of the surface) will be at the origin of the coordinate system. This is not necessarily true. The location of the origin of the coordinate system can have a profound effect on the appearance of the measured profile and on the values of the coefficients in the polynomial fit. A not-so-trivial example of coordinate system origin effects is shown in Fig. 2. Both frames are simulated profiles of exactly the same surface. The origin of the local coordinate system in the left frame is at the pole of the surface segment, while the curve in the right-hand frame is being described in a local coordinate system that is translated along the surface $100 \mathrm{~mm}$ to the right of the pole. At first glance, these look like completely different surfaces, but the appearance is merely an artifact of the origin and orientation of the coordinate systems. If a second order polynomial LSF is done to each set of data points, the values of the coefficient of the second order term will be identical, but the tilt term (first order coefficient) will be non-zero for the right-hand curve.
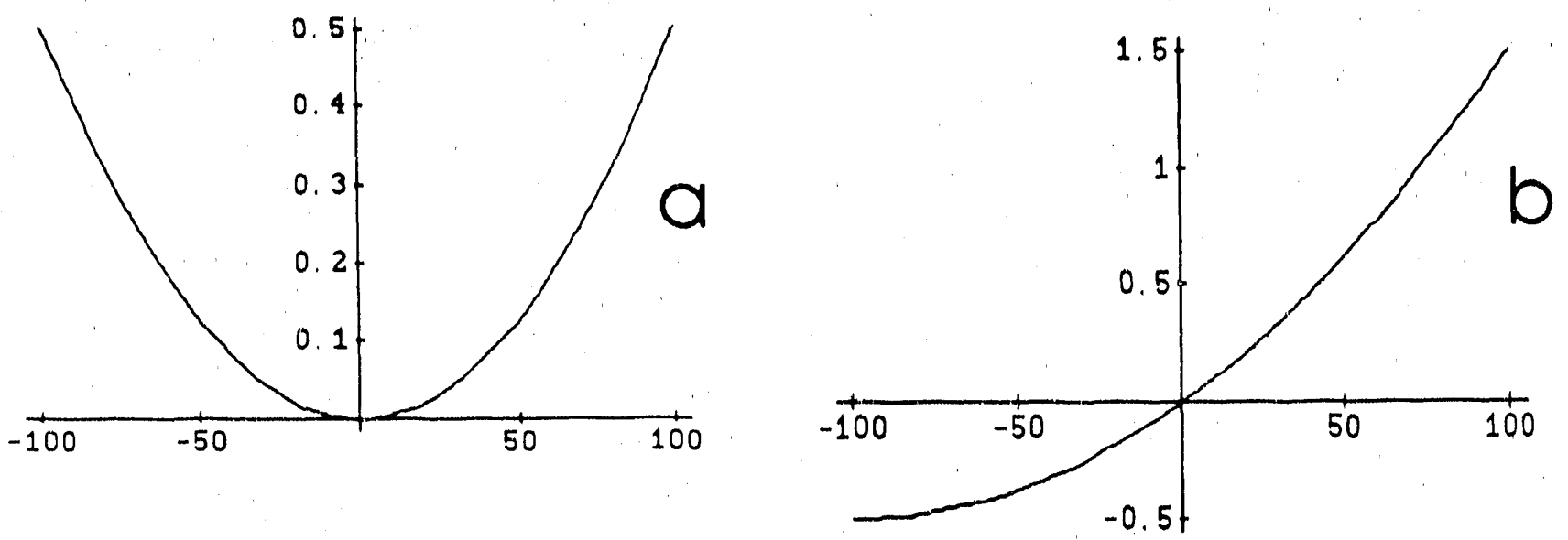

Figure 2.) Appearances are deceiving. Profiles of the same surface, a sphere with $R=$ const, as seen in two different coordinate systems. The system in (b) is shifted $100 \mathrm{~mm}$ to the right of (a). It is difficult to tell that these have the same radius value.

The previous paragraph was an illustration to show that appearances can be deceiving in viewing profiler data. In the special case of the spherical surface, the value of the second order coefficient in the LSF is independent of the measurement geometry, the location of the pole of the surface, and the length of the data set. Thus, the same radius of curvature value is extracted from any measurement. The situation is not quite so simple for higher order 3 urfaces, e.g., ellipsoids. The instantaneous curvature of an ellipsoid is not constant, which requires that the third order term be included in the description of the surface profile in the local coordinate system. The racilus of curvature derived from the third order LSF now depends on the origin of the coordinate system and on the rotation angle of the local system with respect to the pole. 
From the viewpoint of the profiling specialis, ellipsoids are difficult to analyze, not because they are any more difficult to test than a sphere, but because there are numerous ways of defining the section of the ellipsold to be tested. The simplest description of an ellipsoid is in the global coordinate system with the origin at the center of the complete figure:

$$
\frac{x^{2}}{a^{2}}+\frac{z^{2}}{b^{2}}=1
$$

Here there are only two parameters that describe the ellipse: $a$, the semi-major axis, and $b$, the semi-minor axis. In order to completely specify the local segment of the ellipse that is to be tested, we need one more piece of information, ustally the angle of incidence at the pole, $\theta$. We could also be given the parameters of the ellipse in terms of the eccentricity, conic constant, primary and secondary focal lengths, vertex radius, or various combinations of all of the above. We prefer to work with the $[a, b, \theta]$ parameter set and convert all the other combinations into this set as early as possible.

The data analysis process for an ellipse is to start with the description of the surface in the global coordinate system as in Eq. 2, then translate and rotate the surface into the local coordinate system with origin at the pole of the surface. If we do the coordinate transformation in Eq. 2 and then solve for the surface height, $Z_{2}$, as a function of $X_{2}$, we get a polynomial whose coefficients are complicated functions of $a, b$, and $\theta$. This particular transformation procedure results in a polynomial expression for $Z_{2}\left(X_{2}\right)$ with the 0 th and 1 st order coefficients equal to zero. Once the ideal surface is expressed as an analytic function in the local coordinates, the measured data points can be compared to the inalytic function by looking at the differences:

$$
\Delta Z=Z_{m e \omega s}-Z_{2}
$$

This data set describes the departure of the measured surface from the ideal, i.e., it is the residual surface for the ellipse. It should be noted that extracting the values of the three ellipse parameters out of a set of measured data is rather difficult and of dubious value because it involves non-linear regression of complicated functions of the parameters. It is easy to analyze the departure of the measured surface from the ideal ellipse, but difficult to determine what are the best-fit ellipse parameters to the data set.

An example of the analysis procedure used with ellipsoids is iilustrated with results from the measurement of an old mirror which was used in an SX-700 monochromator[3]. The parameters of this mirror are:

$$
\begin{aligned}
& a=26,062.5 \mathrm{~mm} \\
& b=249.5 \mathrm{~mm} \\
& \theta=88 \text { degrees (2 deg grazing incidence). }
\end{aligned}
$$

The full ellipse is shown in Fig. 3a, illustrating the extreme eccentricity of this surface. In fact, the vertical scale is expanded by a factor of ten to show that the shape really is an ellipse and not a straight line. This shape corresponds to the view of the surface in the global coordinate system of Eq. 2. If we perform the required coordinate rotation and translation to the pole of the surface, we obtain the view in Fig. 3b showing the lower right quadrant of the surface. If we now zoom in and take a closer look at the $250 \mathrm{~mm}$ segment of the surface about the pole, we get the view in Fig. 3c. The ideal surface has about a 130 micrometer sag over the $240 \mathrm{~mm}$ clear aperture, and a slight asymmetry is noted between the left and right sides, as the right side curves up faster than the left. The analytic expression for this curve in the local system is given by

$$
Z_{2}\left(X_{2}\right)=8.8955(-6) x^{2}+4.3581(-\cdots 9) x^{3}+\ldots
$$

There are no piston and tilt terms in the analytic expression. 


\section{a Global Coords}

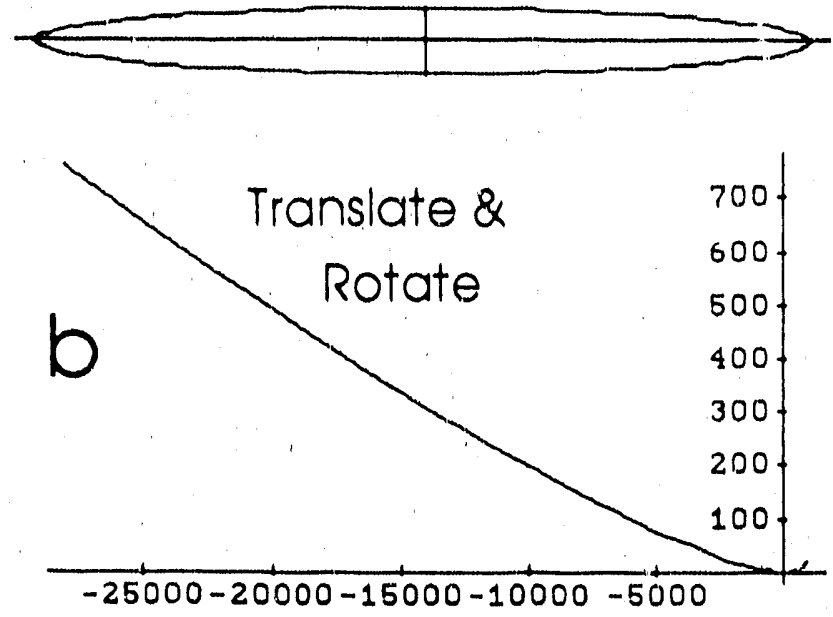

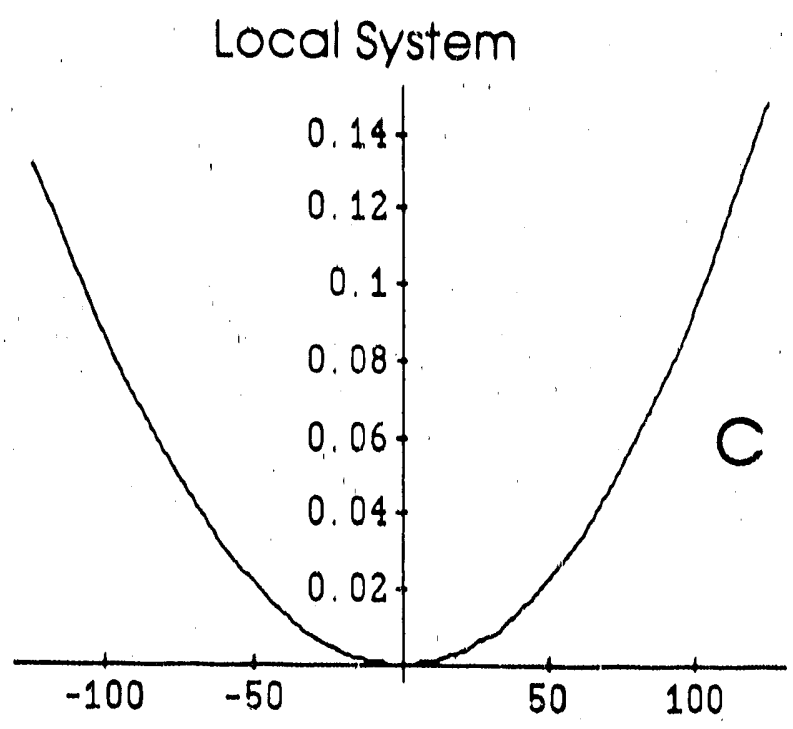

Figure 3.) Transformation from global to local coordinate systems for an off-axis ellipsoid with $a=$ $26,062.5 \mathrm{~mm}, \mathrm{~b}=249.5 \mathrm{~mm}$ and $\Theta=22^{\circ}$ Dimensions in $\mathrm{mm}$. Measurements are to be made over $240 \mathrm{~mm}$ length of the surface. The analytic function of Eq. (4) plotted in (c) has no piston and tilt terms after the coordinate transformation.

Equation (3) is the analytic expression of the ideal ellipsoidal surface, but it is not what the profiler sees. In order to understand the viewpoint of the profiler, let us perform a mathematically allowed, but intuitively irrelevant, operation on the analytic function: do a second order LSF to the above equation to see what is the departure from the best-fit parabola (a parabola is equivalent to a circle over this range of $x$-values). If we do the fit, the result is:

$$
Z_{f i t}=-5.7665(-5)+4.1384(-5) x+8.9321(-6) x^{2}
$$

Comparing Eq. 5 to Eq. 4, we see that the fit equation now has piston and tilt terms, and the curvature coefficient differs slightly between them. What we have done is to force all of the terms above second order in the analytic expression to appear in the first three terms in the LSF expression. Now we can subtract the LSF expression from the analytic equation to get the expression for the departure of the surface from the best-fit parabola:

$$
\left(Z_{2}-Z_{f i t}\right)=5.7665-4.1384(-5) x-3.6615(-8) x^{2}+4.3581(-9) x^{3}+\ldots
$$

Note that the third order term is unchanged by removal of the best-fit parabola. It contains the information about the ellipse. A plot of the residual function of Eq. 6 is shown in Fig. 4. This shows the amount of the third term in the departure of the ellipse segment from a parabola or circle. 


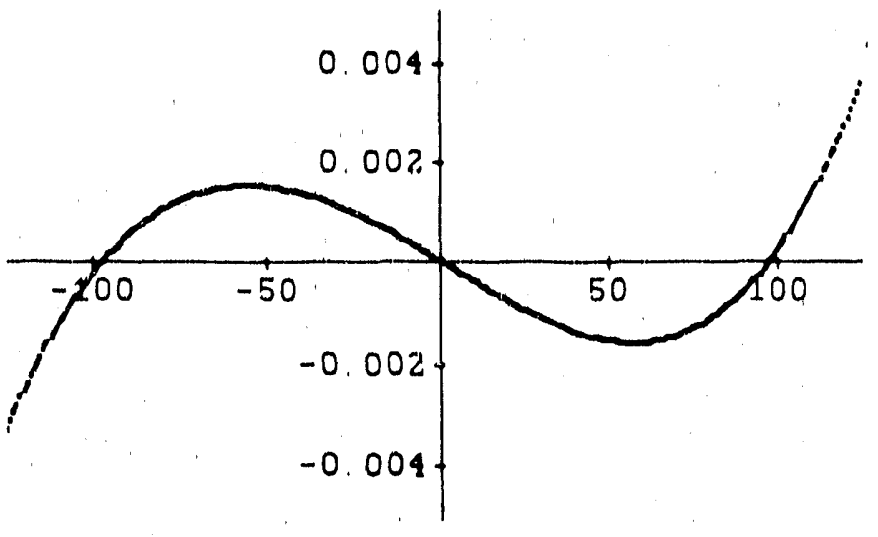

Figure 4.) Residual function after second order LSF to the analytic tunction in Eq. (4). The 2nd order fit adds lower-order terms.

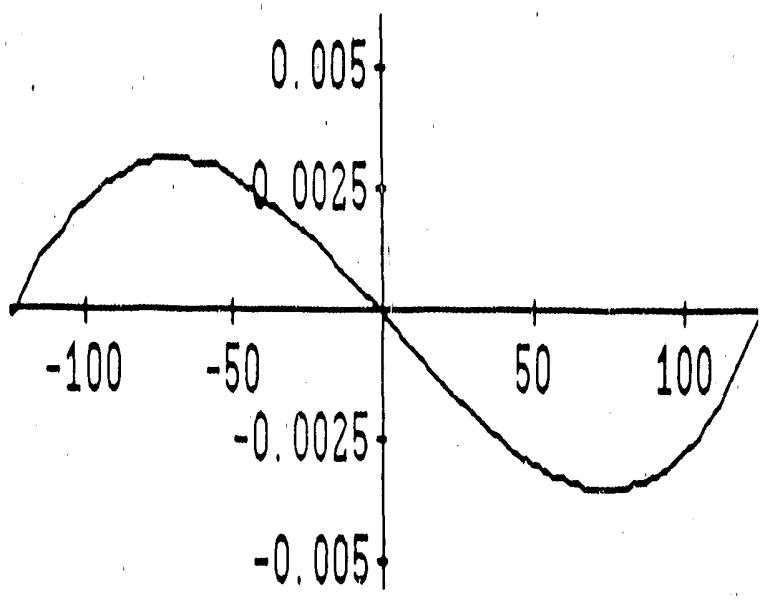

Figure 5.) Predicted view of ellipsold by LTP after detrending piston, tilt, and curvature from slope function. Endpoints are constrained to zero.

We now need to relate this analysis to the actual measurements of the ellipsoidal surface by the LTP. A subtlety of the LTP that affects the data presentation is the fact that it measures the slope of the surface first: the height profile is subsequently computed by a Fourier transform integration in frequency space. We usually remove surface curvature by doing a first order detrend to the raw slope data and then integrating to get the height. If we remove a first order polynomial from the raw slope data and then integrate to get the detrended height profile, we get a curve that is equivalent to a second order detrend of the height with the endpoints constrained to zero. If we simulate this process, starting with the derivative of the analytic function in Eq. 3, we predict that the measured profiler data should look as in Fig. 5. The endpoints are constrained to zero with respect to Fig. 4, and the peak-to-valley departure from a parabola is on the order of $7 \mu \mathrm{m}$ over the $240 \mathrm{~mm}$ measured surface length. The actual measured data from one of the scans on the SX-700 mirror is shown in Fig. 6. The left column is slone and the right column is the corresponding height profile. We see from the Detrend 0 data in the top row that the trid change in slope from one end of the scan to the other is about 4500 microradians, which corresponds to a sag or about $130 \mu \mathrm{m}$. The Detrend 1 results are to be compared to the analytic curve in Fig. 5: the results are nearly identical $-7 \mu \mathrm{m}$ peak-to-valley departure from a parabola. The Detrend 2 results at the bottom in Fig. 6 show the departure of the measured ellipse from the ideal ellipse. The RMS height error for this scan is $0.68 \mu \mathrm{m}$ over the clear aperture and the RMS slope error computed directly from the residual slope data is $18.1 \mu \mathrm{rad}(3.7 \mathrm{arc} \mathrm{sec})$. The figure ard slope errors in this early mirror were significant contributors to degraded resolution in the SX-700 monochromator. Subsequent mirrors with sub-arc second slope errors have improved the resolution of the monochromator significantly[4].

\section{ERROR BARS}

In trying to extract meaningful surface information out of profiler data or any figure measuring device, the questions remain: What are the error bars on the measurement? How significant is the measured profile? How repeatable is the measurement? All of these concerns are related to establishing how well the measurement represents the "true" surface. We can make measurements as presented above, but we don't yet know how significant they are because we don't know what the error bar looks like. Error in LTP measurements arise from several sources, which can be categorized as either noise sources or as systematic error sources. Noist sources produce random variations in the value of the slope function. We can separate the noise sources into high frequency and how frequency sources. Some high frequency sources include air slide pitch angle jitter (porpoising), atmospheric turbulence, detector noise and $A / D$ resolution. Low frequency noise, with a time constant greater than about one minute, arises from thermal and mechanical instabilities in the machine and its environment. A significant source of noise that spans both frequency domains is the inherent pointing stability of the laser source. 


\section{Slope}
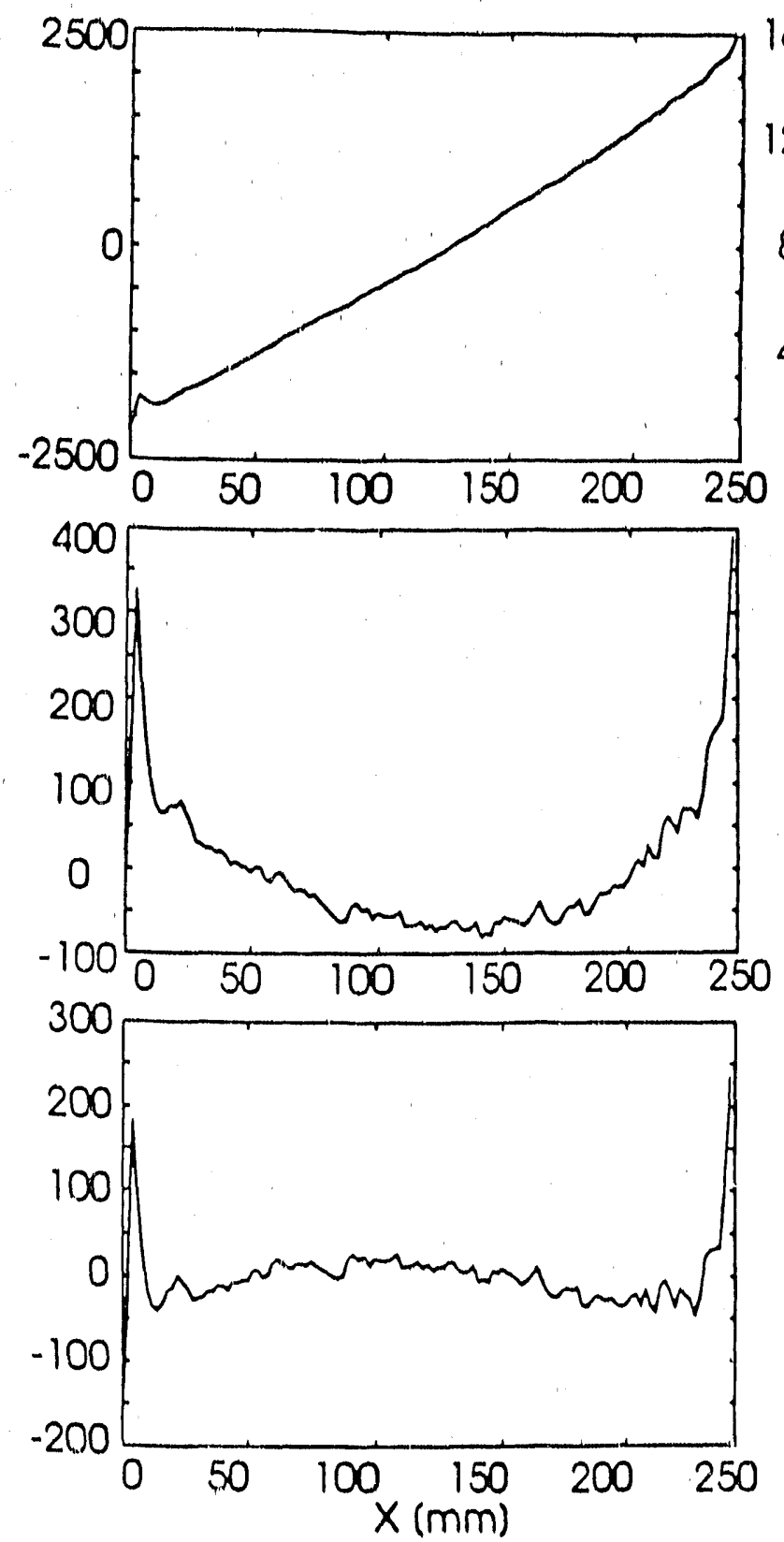

Height
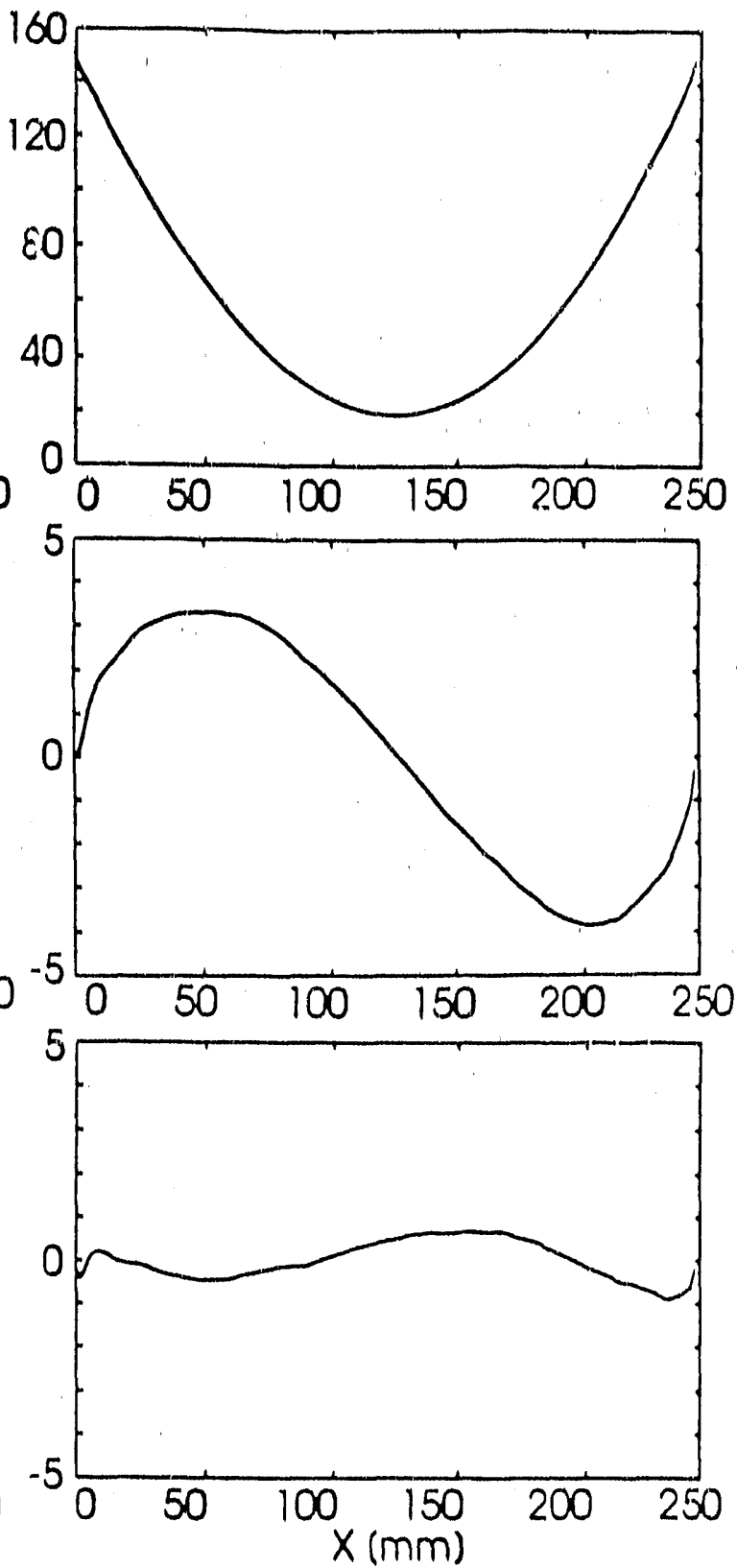

(a)

(b)

Figure 6.) Actual LTP measurements on SX-700 ellipsoldal mirror. Vertical slope axis values are microradians; vertical height axis values are micrometers. (a) Detrend 0 of slope removes piston and tilt from height. (b) Detrend 1 removes curvature, showing residual 3rd order height term. Compare to predicted height in Figure 5. (c) Detrend 2 residual shows departure of surface from the ideal ellipsold. RMS $\sigma_{z}=0.68 \mu \mathrm{m}$; RMS slope error is $18.1 \mu \mathrm{rad}(3.7 \operatorname{arc~sec})$ over $225 \mathrm{~mm}$ clear aperture. 
High frequency noise sources result in a random variation in the slope function, which can be characterized by an RMS slope error value, $\sigma_{\mathrm{m}}$. The RMS slope error can be estimated by having the LTP take a data set without moving between points. We call this a "stabllity scan". The resultant slope error value contains contributions from all noisc sources except those produced by ait slide motion, e.g. porpoising. Typical $\sigma_{\mathrm{m}}$ values for the original LTP system are in the range of 500 to 800 nanoradians per point. For a step size of $1 \mathrm{~mm}$, this corresponds to a height fluctuation of between 5 and 8 Angstroms per puint. In order to compute the size of the error bar for a finite size data record, one must consider the nature of the slope-to-height conversion process. We can compute the power spectral density function of the derived height proflle and then integrate over the frequency range of interest to compute the error bar in real space. The expression for the LTP noise spectrum is given by:

$$
S\left(f_{m}\right)=\frac{D^{3} \sigma_{m}^{2}}{2} \cdot \frac{1}{\sin ^{2}\left(\pi D f_{m}\right)}
$$

where $f_{m}=m /(N D)$ is the surface spatial frequency and $m$ is the index value corresponding to the harmonic of the fundamental frequency $1 /(N D)=1 / L$, which is the inverse of the trace length. The Nyquist frequency for this sampled dinta corresponds to $m=N / 2$, beyond which the power spectrum is not defined. For a trace length of 1000$) \mathrm{mm}$ at a sampling rate of $1 \mathrm{~mm}$ per point, we would have 500 points in the spectruin up to a cutoff frequency corresponding to a $2 \mathrm{~mm}$ spatial period. The high frequency asymptote of Eq. 7 approaches $\left(D^{3} \sigma_{m}{ }^{2}\right) / 2$ as the value of the sine function approaches unity at the Nyquist frequency. We can determine the value of the slope error directly by computing the power spectrum of a stablity stan and observing the value of the high frequency noise. This is illustrated in Fig. 7 for two stability scans. The analytic spectrum is computed from Eq. 7 using $\sigma_{m}=700$ nrads, which is the approximate value of the high frequency asymptote in both data sets. At low frequency, Eq. 7 becomes:

$$
S(f)=\frac{D \sigma_{m}^{2}}{2 \pi^{2}} \cdot \frac{1}{f^{2}}
$$

At low frequency, the noise spectrum increases as $1 / f^{2}$. The integration process in converting from slope the height causes the random slope noise to appear as $1 / f_{2}$ noise in the height. This has the effect of incraasing to size of the error bar as the length of the trace increases. If we integrate the area under the noise spectrum, we compute from the analytic expression, for large $N$, an expression for the helght profile error bar:

$$
\sigma_{z}=\sqrt{\frac{N}{12}} \cdot D \cdot \sigma_{m}
$$

This is the error bar that characterizes the quality of the measured surface profile. Typical values of the height uncertainty are calculated in Table I for various values of $N$, assuming a sampling distance of $D=1 \mathrm{~mm}$ and a slope noise value of $a_{\mathrm{m}}=800 \mathrm{nrad}$. One can see from the last column that the absolute accuracy of the measurement is below the level of $\lambda / 100$ for trace lengths up to $500 \mathrm{~mm}$.

The numbers in the table reflect performance of the instrument assuming that high frequency random noise is the only noise source. Other noise sources contribute to excess low frequency noise, which is evident in Fig. 7 as an increase above the $1 / f^{2}$ asymptote at low frequencies. The two measured data sets correspond to two different integration times and interva!s between data points. The integration times differ by a factor of eight between the two curves. As the integration time increases, the low frequency sources begin to distort the gross shape of the surface. The RMS height error computed by integrating the area under the curves in the low frequency range corresponding to $1090 \mathrm{~mm} 10$ $100 \mathrm{~mm}$ spatial periods is $7.7 \mathrm{~nm}$ for the fast scan and $27.5 \mathrm{~nm}$ for the slow scan. The RMS numbers over the high frequency range, corresponding to periods shorter than $100 \mathrm{~mm}$, are nearly the same, $0.16 \mathrm{~nm}$ and $(0.19 \mathrm{~nm}$. The nextgeneration LTP currently under construction will have a significantly Improved noise performance. One source of low 


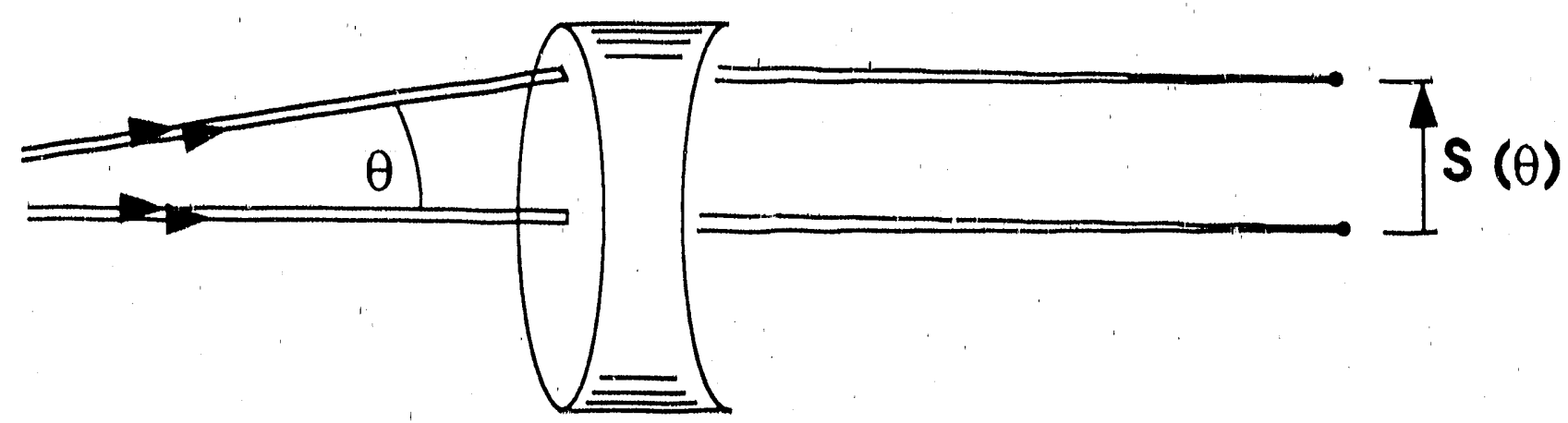

Figure 8.) Sketch of the function of the LTP optical sysiem. Beams reflected from the test surface at an angle theta from the normal are mapped into lateral position on the detector. Deviations from a lincar mapping function arise from systematic aberrations.

\section{SYSTEMATIC ERROR EFFECTS}

Any metrology system has to deal with systematic error sources. The LTP is unique in the way it corrects for pitch angle errors caused by the sag of the air bearing beam as the optical head traverses over it. Another source of error is found in the aberrations in the Fourier transform and relay optics that bring the beam onto the detector. The optical syster. should perform as an ideal linear angle-to-position converter, as lllustrated in Fig. 8. The laser beam pairs reflected off of the test surface re-enter the optical head at various angles, $\theta$, which depend on the local slope of the surface. The optical system should map the angles linearly onto the detector, so that the position of the beam centrold becomes a linear function of $\theta$. There is, however, a slight amount of aberration so that the angle-to-distance transformation is not perfect. Usually this aberration is negligible. However, when we are testing high-quality, short radius spheres the change in slope across the surface can exceed the \pm 4.5 mrad acceptance angle in the present LTP s;item. In this case we should expect to see some effects from system aberration. The opportunity to evaluate the system aberration arose during the testing of a high-quality, nearly spherical surface which was to be used in a normal incidence multilayer-coated $x$-ray telescope system[6].

The mirror that was tested was in the shape of an on-axis ellipsoid with an eccentricity of 0.95 and a vertex radius of almost 4 meters. The surface was very nearly a paraboloid. In order to achieve sub-arc second resolution at $\lambda=$ 63 Angstroms, the surface finish and surface figure quality had to be quite high. The surface figure quality was measured by the manufacturers to be in the range of $1 / 100$ wave in visible light. An attempt was made to measure the profile of the surface with the LTP. Because of the rather short radius of curvature, only $33 \mathrm{~mm}$ of the surface could be profiled at a time. The surface curvature exceeded the $\pm 4.5 \mathrm{mrad}$ limit for traces longer than $33 \mathrm{~mm}$. We expected to see departures from a second order curve on the order of $3 \mathrm{~nm}$ over the $33 \mathrm{~mm}$ trace length arising from the slight ollipsoidal nature of the mirror. Instead, we saw departures on the order of $30-40 \mathrm{~nm}$ from a parabola in each scan. A typical set of scans is shown in Fig. 9. Each scan looked remarkably like all others, although we made radial scans at nine different locations in both the positive and negative radial directions. We suspected that the mirror quality might be high enough to allow us to see effects of system aberration. Assuming that the mirror surface figure was indeed better than our optical system, we generated a reference file by averaging all nine data scans together. The result of doing a Detrend 1 on the reference slope function is shown in Fig. 10a. The corresponding helght profile in Fig. 10b shows that the predominant error produces a fourth order height variation across the field of view with just a bit of lower-order odd aberration to skew the profile slightly. The peak-to-valley error is about $\pm 40 \mathrm{~nm}$, corresponding to an RMS aberration value of about $25 \mathrm{~nm}$. In terms of visible wavelengths, the system aberration is about $1 / 25$ wave. 

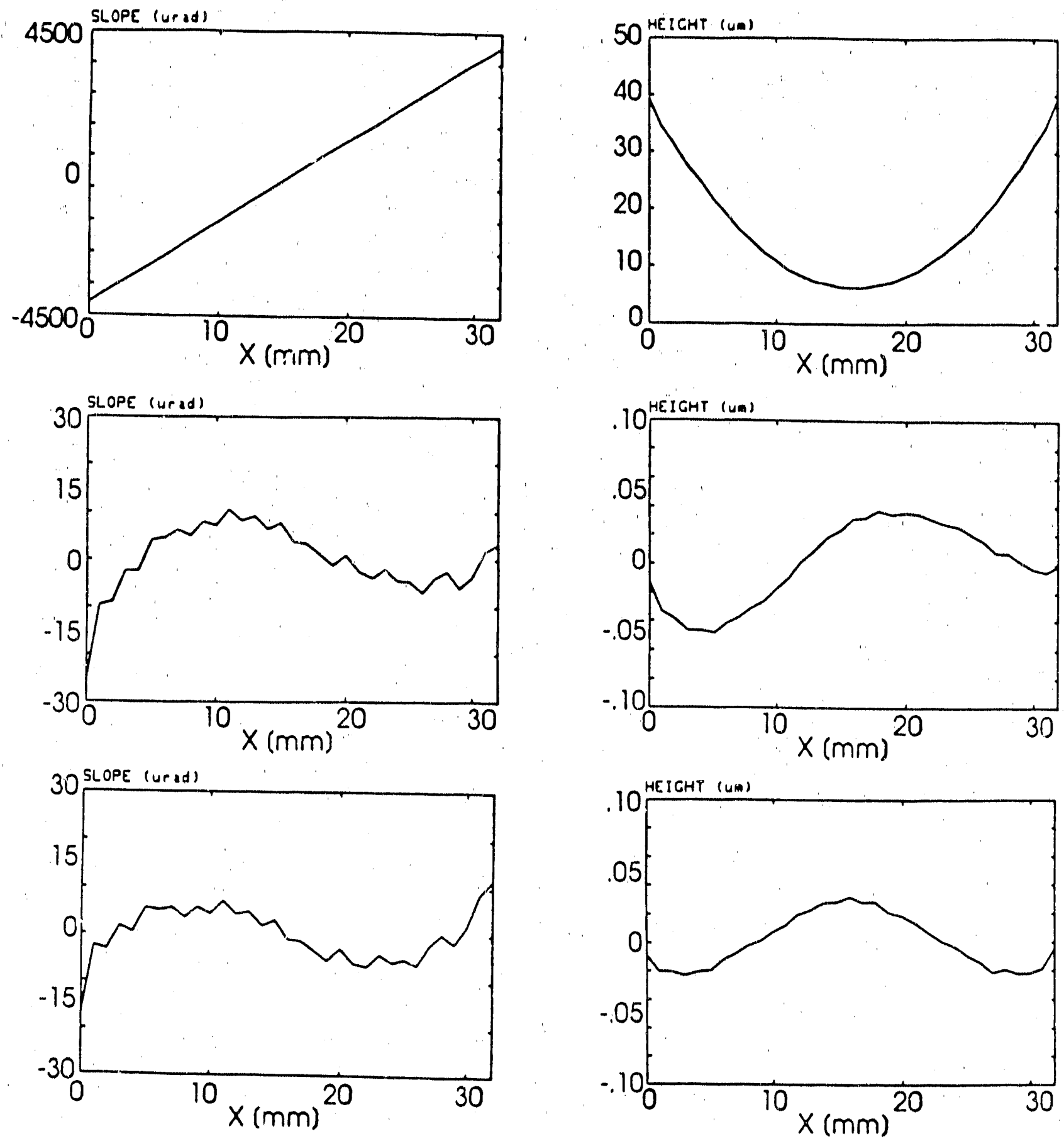

Figure 9.) Single $33 \mathrm{~mm}$ scan across segment of a high quality 4 meter radius mirror. (Top) Raw slope and height with piston and tilt removed. Slope function extends across entire detector range of nearly 9 mrads. (Middle) Detrend $1=$ curvature removed from height. Magnitude of residual is about 10 times greater than expected from asphericity of the surface. (Bottom) Detrend $2=$ third order height term removed, showing residual aberration. 

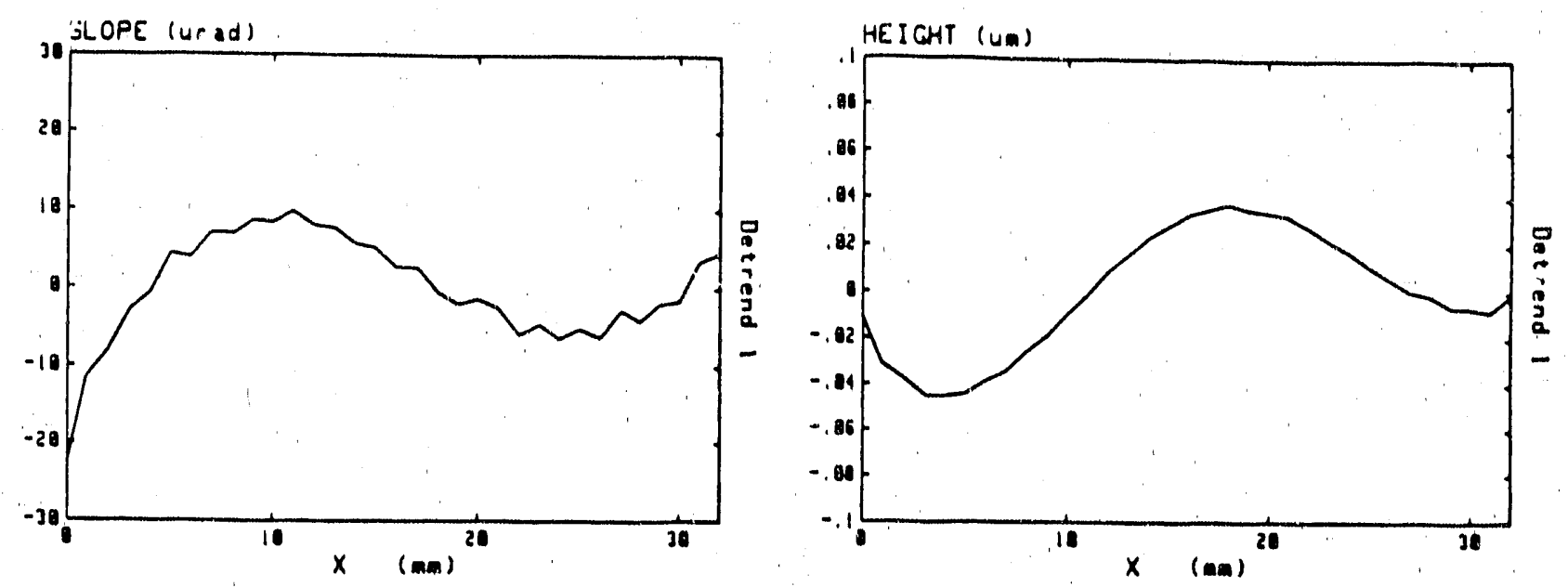

Figure 10.) Reference function generated from average of 9 scans, shown here after curvature is removed to illustrate the magnitude of the correction - about $40 \mathrm{~nm}$ peak-to-valley or about $1 / 25 \mathrm{th}$ of a wavelength of visible light RMS.
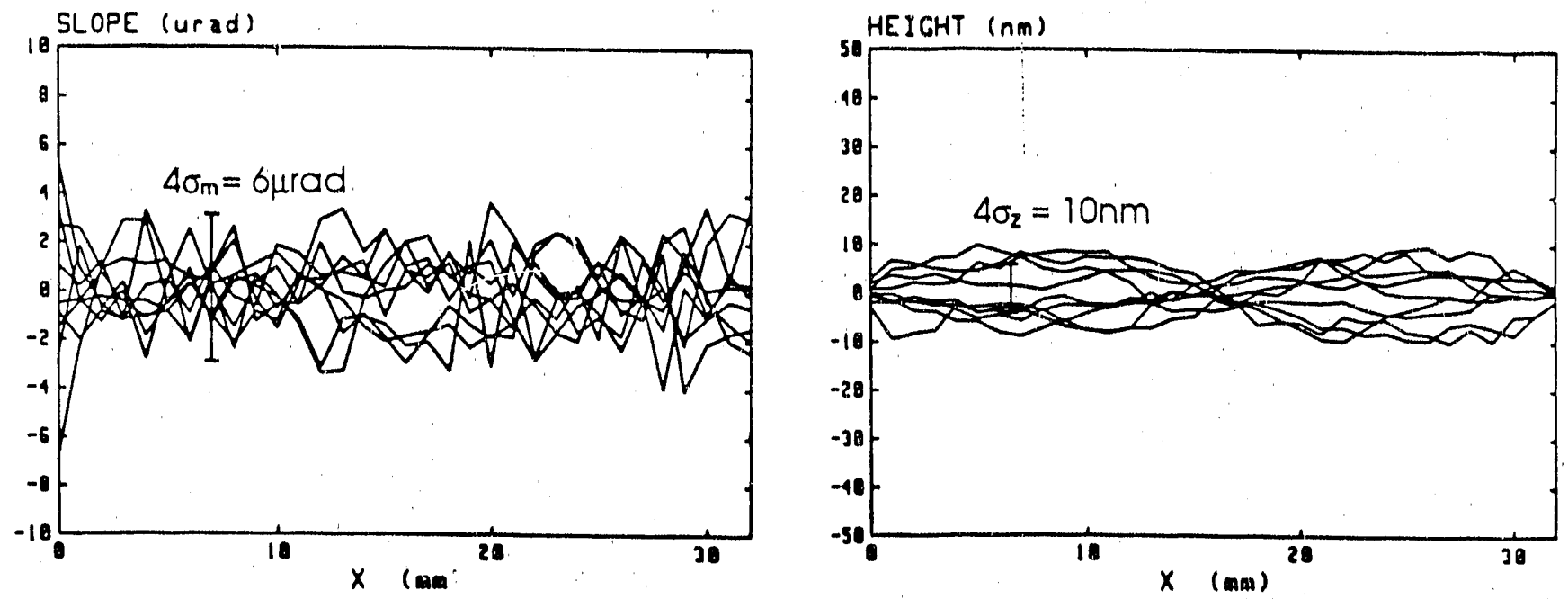

Figure 11.) Residual slope and height profiles for each of the 9 scans on the high-quality mirror. Height error bars are computed from the 4-sigma experimental slope error bar using the analytic expression in Eq. (9). The 1-sigma height error of $2.5 \mathrm{~nm}$ corresponds to $1 / 250 \mathrm{th}$ of a wavelength of visible light.

Knowing what the system aberration looks like, we can now go back and correct each individual data set and plot the results. Figure 11a shows the results of subtracting the reference slope function from each raw slope data set and then doing a Detrend 0 on the result to give it a zero-mean slope function (equivalent to removing piston and tilt from the height profile). The corresponding height profiles are shown superimposed in Fig. 11b. An eyeball estimate of the 
slope error of the corrected slope functions returns an estimate of the $4-\sigma$ value of about $6 \mu$ rads, or a value for $\sigma_{\mathrm{m}}$ $=1.5 \mu \mathrm{rad}$. The corresponding error bar for a trace length of $33 \mathrm{~mm}$ according to Eq. 9 is $\sigma_{z}=2.5 \mathrm{~nm}$ or the $4-\sigma$ value is $10 \mathrm{~nm}$. The $4-\sigma$ error bars are shown on the plots. Static noise analysis for an assumed slope jitter of $0.8 \mu$ rad per point gives a value for $\sigma_{2}$ of $1.0 \mathrm{~nm}$. Owing to the roundabout nature of the reference file generation, the correspondence hetween measurement residuals and prediction is quite good. The experimental error bar of $2.5 \mathrm{~nm}$ corresponds to a $1 / 250$ wave error after correcting the measurements for the system aberration. We attempted to make an independent measurement of the system aberration function by reflecting the probe beams off of a flat mirror mounted on a lever arm one meter long, but leadscrew errors of \pm 30 micrometers in all of the motorized micrometers that were available to us prevented us from obtaining a usable angle calibration. The best calibration was accomplished with the telescope mirror itself.

\section{CONCLUSIONS}

There are many issues involved in measuring the absolute shape of an optical surface by profilometry. Some are simply a matter of viewpoint, as in detrending over a particular range to enhance or hide certain features. Others involve knowledge of the fundamental instrumental noise limits that allow one to determine the size of the error bar on a particular measurement. It is that error bar that determines the envelope around the measured data points within which the true surface can be found. We have illustrated the detrending process with some typical examples of surfaces that were measured by the Long Trace Profiler. Extracting low-order figure parameters, such as radius of curvature, from LTP measurements must be done with the awareness that different values for certain parameters will result from the least-squares fitting process if one uses the raw slope data or the processed height data. It is important to specify the particular data analysis path to be used in determining figure parameters. We have also illustrated our knowledge of the noise limits of the present version of the LTP. For trace lengths of several hundred millimeters, we can place error bars around the measurement that are better than $1 / 100$ of a wave of visible light. The next generation LTP system will have superior noise performance and will be far less susceptible to low frequency noise sources, such as thermal drifting and laser pointing instability.

\section{ACKNOWLEDGEMENTS}

The authors acknowledge the assistance of Mr. Frank Cooke, Dr. William Peatman, Dr. Helmuth Petersen, and Dr. Eberhard Spiller in providing us with mirrors used in the present analysis.

\section{REFERENCES}

1. P. Z. Takacs, S.-C. K. Feng, E. L. Church, S.-N. Qian, and W.-M. Liu, "Long Trace Profile Measurements on Cylindrical Aspheres," Advances in Fabrication and Metrology For Optics and Large Optics, Jones B. Arnold, Robert E. Parks, eds., Proc. SPIE 966, 354-364 (1989).

2. P. Z. Takacs, K. Furenlid, R. De Biasse, and E. L. Church, "Surface Topography Measurements Over the 1 Meter to 10 Micrometer Spatial Period Bandwidth," Surface Characterization and Testing II, J. E. Grewenkamp, M. Young, eds., Proc. SPIE 1164, 203-211 (1989).

3. Helmuth Petersen, "The Plane Grating and Elliptical Mirror: A New Optical Configuration for Monochromators," Optics Communications, 40, 402-406 (1982).

4. Helmuth Petersen, "Installation und Tests des neuen sub-arc sec-Fokussierspiegels für den SX700/1," BESSY Technischer Bericht TB136/88, Dezember 1988. 
5. Nicholas M. Sampas and Dana Z. Anderson, "Stabilization of Laser Beam Alignment to an Optical Resonator by Heterodyne Detection of Off-axis Modes;" Appliad Optics, 29, 394-403 (1990).

6. E. Spiller, R. McCorkle, J. Wilczynski, L. Golub, G. Nystrom, P. Takacs, and C. Welch, "Imaging Performance and Tests of Soft X-ray Telescopes," X-Ray/EUV Optics for Astronomy _ Microscopy Polarimetry and Projection Lithography, R. B. Hoover, ed., Proc. SPIE, 1343, in press. 

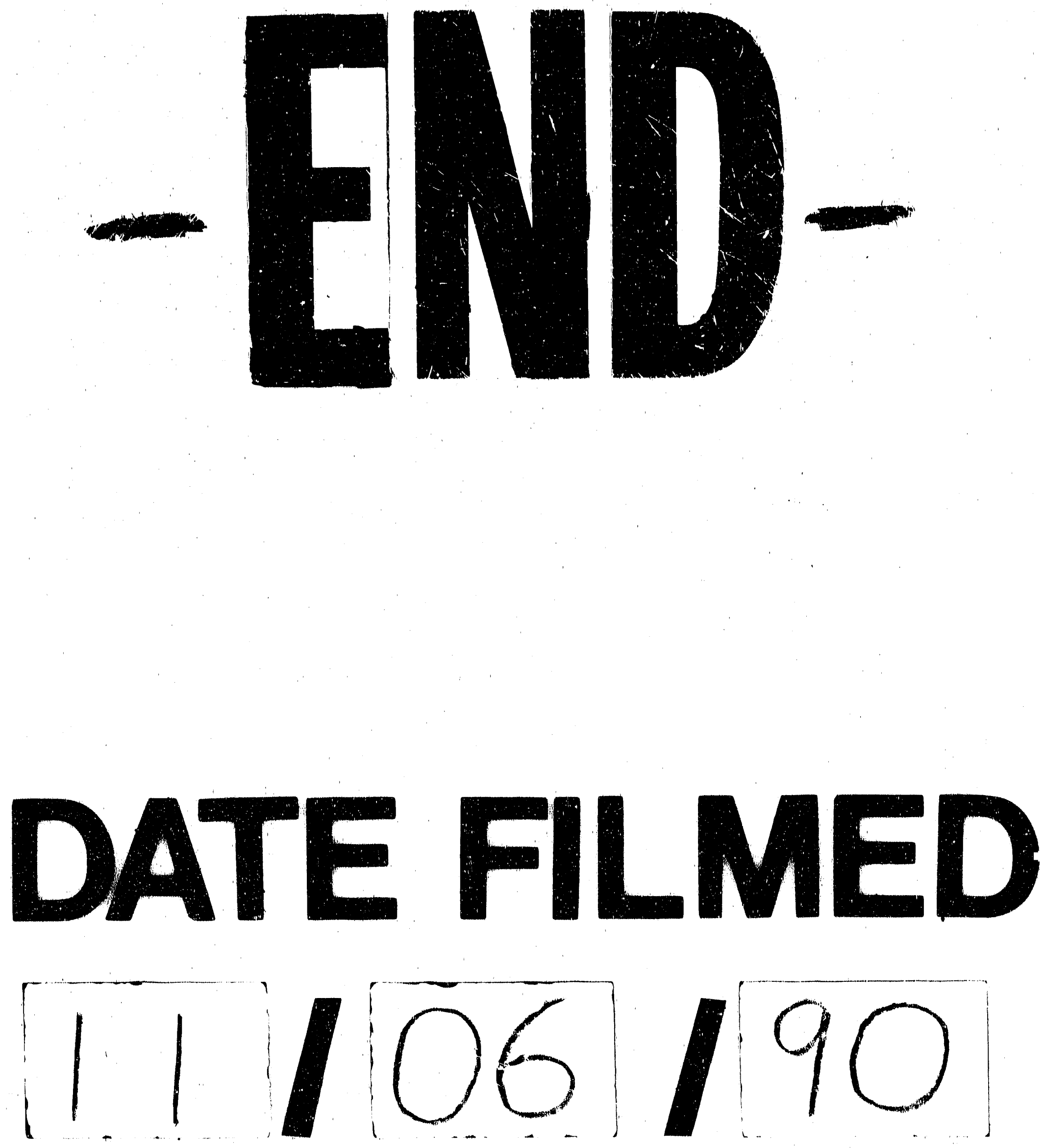
